\title{
Modelling selective activation of small myelinated nerve fibres using a monopolar point electrode
}

\author{
$\begin{array}{llll}\text { N. J. M. Rijkhoff } & \text { J. Holsheimer } & \text { F. M. J. Debruyne } & \text { H. Wijkstra }\end{array}$ \\ 'Department of Urology, University Hospital Nijmegen, PO Box 9101, $6500 \mathrm{HB}$ Nijmegen, \\ The Netherlands \\ ${ }^{2}$ Biomedical Engineering Division, Department of Electrical Engineering, University of Twente, \\ The Netherlands
}

\begin{abstract}
The aim of this study is to investigate theoretically the possibility for activation of small myelinated nerve fibres without activating larger ones when stimulating a nerve fibre bundle using a monopolar point electrode. Therefore, the sensitivity of excitation and blocking threshold currents of nerve fibres to fibre diameter, electrode-fibre distance and pulse duration has been simulated by a computer model. A simple infinite, homogeneous volume conductor and a cathodal point source were used in combination with a model representing the electrical properties of a myelinated nerve fibre. The results show that selective activation of small myelinated fibres may be possible in a region at some distance from the electrode.
\end{abstract}

Keywords-Electrical nerve stimulation, Modelling, Selective small fibre activation

Med. \& Biol. Eng. \& Comput., 1995, 33, 762-768

\section{Introduction}

ELECTRICAL STMULATION of nervous tissue using implanted electrodes can be used to induce muscle contraction in patients with an upper motoneuron lesion. The use of this concept to restore lost functions is limited in performance and applications. One of the problems is the inverse recruitment order of nerve fibres compared with physiological recruitment. In physiological muscle contraction, the recruitment of motor units by the CNS is in order of increasing motor unit size (the size principle, HENNEMAN, 1981). As large-diameter nerve fibres need a smaller extracellular stimulus for their activation than smaller fibres (BLAIR and ERLANGER, 1933), electrical stimulation recruits large motor units (driven by large-diameter motoneurons) before smaller ones. This results in poor control of muscle force (SOLOMONOW, 1984). The control of muscle force would be improved by the restoration of physiological recruitment order by primarily activating small-diameter motoneurons.

Another application of selective small fibre activation is in urology. Electrical stimulation of ventral sacral roots to induce urinary bladder emptying results in simultaneous activation of the detrusor and the urethral closure mechanism, leading to little or no voiding (SCFMNDT, 1986). As the detrusor is innervated by fibres of a smaller diameter than the urethral sphincter (SCHALOW and BARTH, 1992), selective activation of the small fibres in the ventral sacral roots would result in

Correspondence should be addressed to Ir. Nico J. M. Rijkhoff First received 18 November 1994 and in final form 20 April 1995

(IFMBE: 1995 selective detrusor activation, and thus better stimulationinduced bladder emptying.

Selective small-fibre activation can be obtained by a combination of excitation of both large- and small-diameter fibres, and blocking, distal to the excitation point, the propagation of the induced action potentials in the larger fibres. Blocking may occur close to an anodal contact due to hyperpolarisation of the nerve membrane. If the nerve membrane is sufficiently hyperpolarised, action potentials cannot pass the hyperpolarised zone and are annihilated. As large-diameter fibres need a smaller extracellular stimulus for blocking than smaller fibres (ACCORNERO et al., 1977; FANG and MORTIMER, 1991a; RIJKHOFF et al., 1994), a selective blockade of the large fibres is possible. It has been shown by computer modelling and in animal experiments that a tripolar electrode configuration can be used to achieve selective smallfibre activation (FANG and MORTIMER, 1991a,b; RIJKHOFF et al., 1993, 1994).

However, the most simple configuration to achieve selective small-fibre activation is monopolar stimulation using a cathodal current. The blockade is caused by hyperpolarisation of the regions of the fibre at some distance from the cathode. This effect is presented by Ranck as the 'anodal surround' effect (RANCK, 1975). Rattay was the first to show this blocking effect in a computer model when simulating the responses of unmyelinated fibres to monopolar stimulation (RATTAY, 1987). The influerice of both fibre diameter and pulse duration on the threshold for blocking has been investigated by Tai and Jiang, but they also used a model of an unmyelinated axon (TAI and JANG, 1994). As beyond $1.5 \mu \mathrm{m}$ all mammalian nerve fibres are myelinated, their results have a limited value. Theoretical results on blocking of myelinated fibres have been 


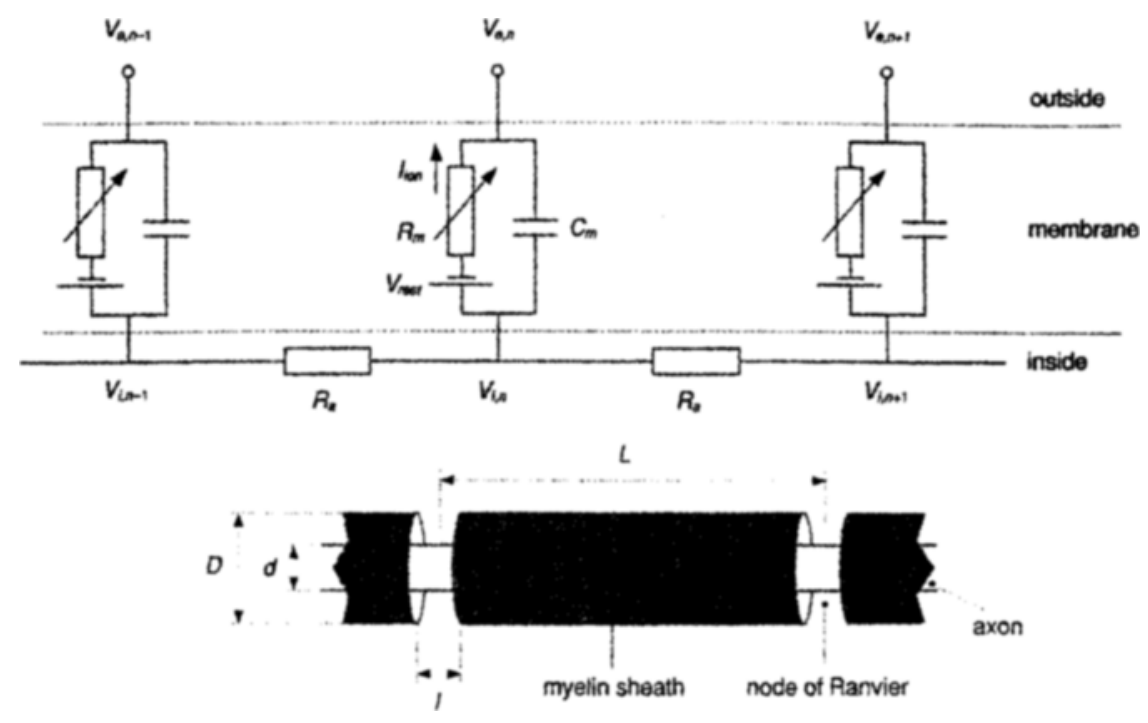

Fig. 1 Electrical network equivalent of myelinated nerve fibre

reported previously (RATTAY, 1989) but those data were only presented as a demonstration of the model capabilities.

In this paper, the possibilities of selective activation of small myelinated nerve fibres using a monopolar cathodal point electrode are investigated using a computer model. In order to obtain an insight into the influence of stimulation parameters on fibre diameter selectively, we have investigated the effect of fibre diameter, cathode-fibre distance and pulse duration on the threshold currents needed for excitation and blocking.

\section{Methods}

The propagation of an action potential in a myelinated nerve fibre is a consequence of the electrical properties of the axon. These electrical properties can be represented by an electrical network, as shown in Fig. 1. The membrane at each node of Ranvier is represented by an electrical circuit consisting of a capacitor $C_{m}$, a voltage source $V_{\text {rest }}$ and a nonlinear resistor $R_{m}$. $R_{a}$ represents the resistance of the axoplasm between two neighbouring nodes of Ranvier. This model was adopted from that of McNeal (MCNEAL, 1976).

To calculate the response of a fibre to an extracellular electric potential field, the following relation was derived between the reduced transmembrane voltage $V_{n}$ $\left(=V_{i, n}-V_{e, n}-V_{\text {rest }}\right)$ of node $n$ and the extracellular potential $V_{e, n}$ at each node of Ranvier:

$$
\begin{array}{r}
\frac{\mathrm{d} V_{n}}{\mathrm{~d} t}+\frac{I_{\text {ian, } n}}{C_{m}}-\frac{1}{R_{a} C_{m}}\left(V_{n-1}-2 V_{n}+V_{n+1}\right) \\
=\frac{1}{R_{a} C_{m}}\left(V_{e, n-1}-2 V_{e, n}+V_{e, n+1}\right)
\end{array}
$$

Assuming that the axon is a cylinder with constant diameter $d_{\text {, }}$ then the intra-axonal resistance $R_{a}$, the nodal membrane capacity $C_{m}$ and the ionic current $I_{i o n, n}$ can be expressed in fibre parameters:

$$
\begin{aligned}
& R_{a}=4 \rho_{a} L / \pi d^{2} \\
& C_{m}=c_{m} \pi d l \\
& I_{\text {ion, } n}=i_{\text {ion,n, } n} \pi d l
\end{aligned}
$$

The symbols and the parameters of the nerve fibre model are summarised in the Appendix. Inserting eqn. 2 into eqn. 1 yields

$$
\begin{array}{r}
\frac{\mathrm{d} V_{n}}{\mathrm{~d} t}+\frac{i_{i o n, n}}{c_{m}}-\frac{\mathrm{d}}{4 \rho_{a} L l c_{m}}\left(V_{n-1}-2 V_{n}+V_{n+1}\right) \\
=\frac{d}{4 \rho_{a} L l c_{m}}\left(V_{e, n-1}-2 V_{e, n}+V_{e, n+1}\right)
\end{array}
$$

The only variable in eqn. 3 that needs a further description is the ionic transmembrane current density $i_{i o n}$ at the nodes of Ranvier. McNeal used the Frankenhaeuser-Huxley equations, derived from a myelinated frog nerve, to describe the dynamic behaviour of the membrane resistance at the nodes of Ranvier (MCNEAL, 1976). The properties of mammalian nerve fibres, however, are different. Most notable is that the voltagedependent potassium channels shown in amphibians are almost absent at the nodes of Ranvier in mammals (CHU et al., 1979; BRISMAR, 1980). Sodium and leakage currents seem to dominate in those membranes. Therefore, the equations of the membrane kinetics have been adapted according to Chiu et $a l$., who derived them from experimental data from myelinated rabbit axons (CHU et al., 1979). All temperature-dependent parameters were scaled to $37^{\circ} \mathrm{C}$ (equations and parameters are described elsewhere; STRUIJK et al., 1992).

For simplicity we assumed that the fibre lies in an infinite homogeneous medium. The fibre is stimulated using a single point source, and so the electrical potential $V_{e}$ at each node can simply be calculated by

$$
V_{e}(r)=\frac{\rho_{e} I}{4 \pi r}
$$

with $r$ the distance between current source and each node, $I$ the stimulation current (cathodal current $I<0$ ) and $\rho_{e}$ the electrical resistivity of the external medium. For $\rho_{e}$ a value of $3 \Omega \mathrm{m}$ was used, which is an average value for nervous tissue (GEDDES and BAKER, 1967).

The initial value of the transmembrane voltage of all nodes $V_{n}(t=0)$ was set at zero. The integration time step was $1.0 \mu$ s. To calculate the excitation and blocking thresholds, the fibres always had 15 nodes with a nonlinear membrane resistance.

\section{Results}

Approximately $100 \mu \mathrm{s}$ after the onset of a rectangular subthreshold stimulus pulse, the transmembrane potential distribution along a fibre becomes constant until the pulse 


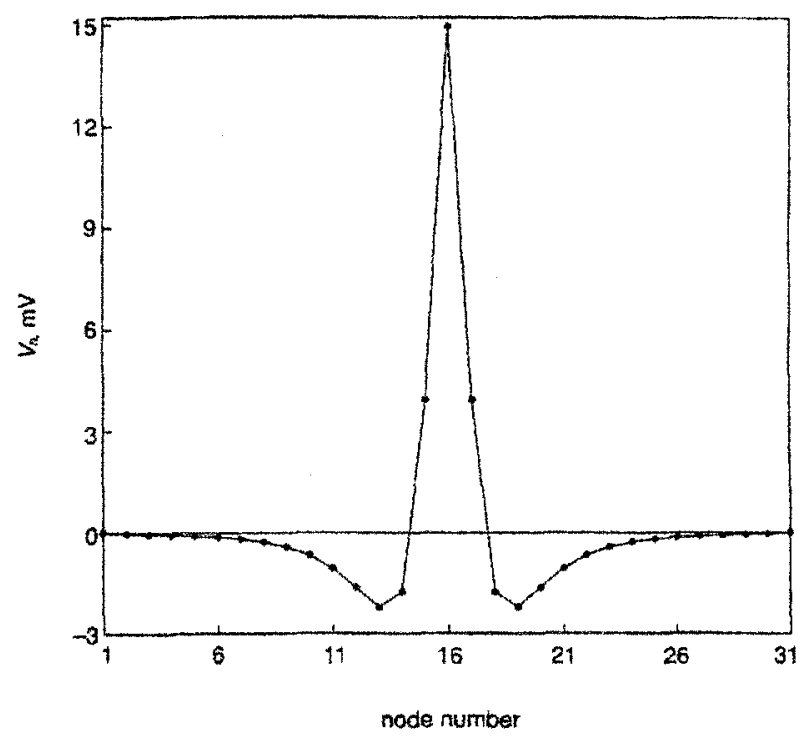

Fig. 2 Reduced transmembrane potential $V_{n}$ (deviation from resting potential) at each node of a $10 \mu \mathrm{m}$ fibre at $1 \mathrm{~mm}$ distance from the cathodal point source at $100 \mu$ s after the start of a $200 \mu s$ square stimulus pulse of $0.22 \mathrm{~mA}$; the point source was above node 16; greatest hyperpolarisation is at node 13 and 19

stops. Fig. 2 shows the transmembrane potential distribution of a $10 \mu \mathrm{m}$ fibre when stimulated by a cathodal point source at $1.0 \mathrm{~mm}$ distance with $0.22 \mathrm{~mA}$ (excitation threshold $0.226 \mathrm{~mA}$ ). The distribution has a typical triphasic shape with a central positive maximum flanked by two negative peaks. At the positive part, the fibre is locally depolarised, whereas at the negative parts the fibre membrane is hyperpolarised. When the membrane depolarisation exceeds the excitation threshold, the fibre is excited and produces an action potential. The action potential then propagates in both directions. However, if the membrane is sufficiently hyperpolarised when the action potential arrives at the hyperpolarised zone, the action potential cannot pass this zone and the propagation is blocked.

Fig. 3 shows the responses of a $10 \mu \mathrm{m}$ myelinated fibre model to a $200 \mu \mathrm{s}$ rectangular pulse with two different amplitudes. At each node, depolarisation is shown with a bar above the line, whereas hyperpolarisation is indicated below the line. Fig. $3 a$ shows the response to a stimulus of $0.35 \mathrm{~mA}$, being above the excitation threshold of $0.226 \mathrm{~mA} .50 \mu \mathrm{s}$ after the beginning of the stimulus (top line) the central node is excited, and with increasing time there is successive excitation of nodes left and right of the centre node, indicating that an action potential propagates in both directions. The action potential propagates through the hyperpolarised regions, indicating that the membrane is not sufficiently hyperpolarised to block the action potential. Fig. $3 b$ shows the response to a stimulus of $1.30 \mathrm{~mA}$, being above the blocking threshold of $1.19 \mathrm{~mA}$. The fibre is again excited at the centre node, but the action potential cannot pass the two zones where the fibre is hyperpolarised. The propagation is blocked and the action potential dies out.

\subsection{Relation between excitationblocking threshold and fibre diameter}

Using a $300 \mu$ s pulse, we calculated the excitation and blocking thresholds for various fibre diameters. As the thresholds are influenced by the relative position of the point source to the nodes of Ranvier (RANCK, 1975; RATTAY, 1987), both excitation and blocking thresholds have been calculated for two different electrode positions:

(a) right above the centre node

(b) at half the intemodal distance from the centre node, both at $1.0 \mathrm{~mm}$ from the fibre.

The results (Fig. 4a) show that the current needed to excite or to block a fibre increases with decreasing fibre diameter. The highest excitation threshold is found with the electrode centred between two nodes. However, the influence of the electrode position relative to the node on the excitation threshold decreases with decreasing fibre diameter. The excitation threshold ratio of position (b) to position $(a)$ decreases from 1.95 to 1.07 when the fibre diameter is reduced from $20 \mu \mathrm{m}$ to $5 \mu \mathrm{m}$.

The influence of electrode position on the blocking thresholds also decreases with decreasing fibre diameter. However, the electrode position that results in the lowest blocking threshold depends on the fibre diameter. For example, the block ratios of position (b) to position (a) for $20 \mu \mathrm{m}, 15 \mu \mathrm{m}$ and $10 \mu \mathrm{m}$ fibre are $1.29,0.87$ and 1.05 , respectively. The
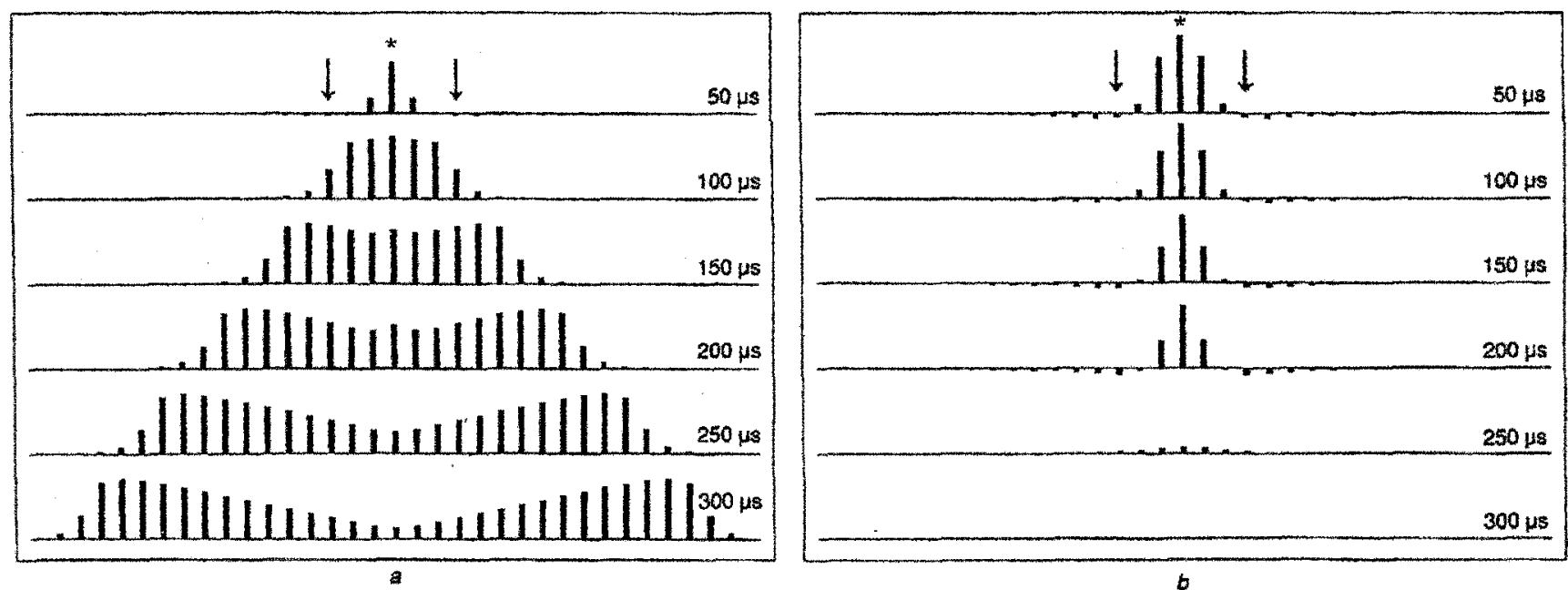

Fig. 3 Electrical response of a 10 um fibre at $1 \mathrm{~mm}$ distance from the cathodal point source to a $200 \mu$ s rectangular stimulus; the nodal potentials (deviation from resting potential) are shown at six intervals of $50 \mu$ after initiation of the pulse; (a) stimulus: 0.35 mA (excitation threshold $0.226 \mathrm{~mA}$ ); (b) stimulus: $1.30 \mathrm{~mA}$ (blocking threshold $1.19 \mathrm{~mA}$ ): * position point source; arrows show the most hyperpolarised nodes (see Fig. 2) 


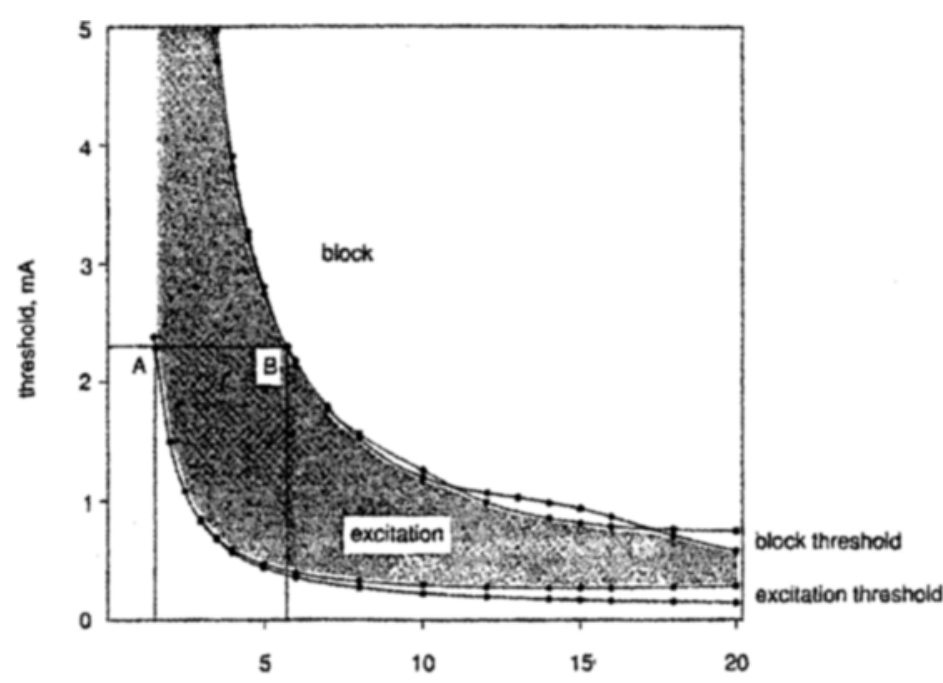

fibre diameter, $\mu \mathrm{m}$

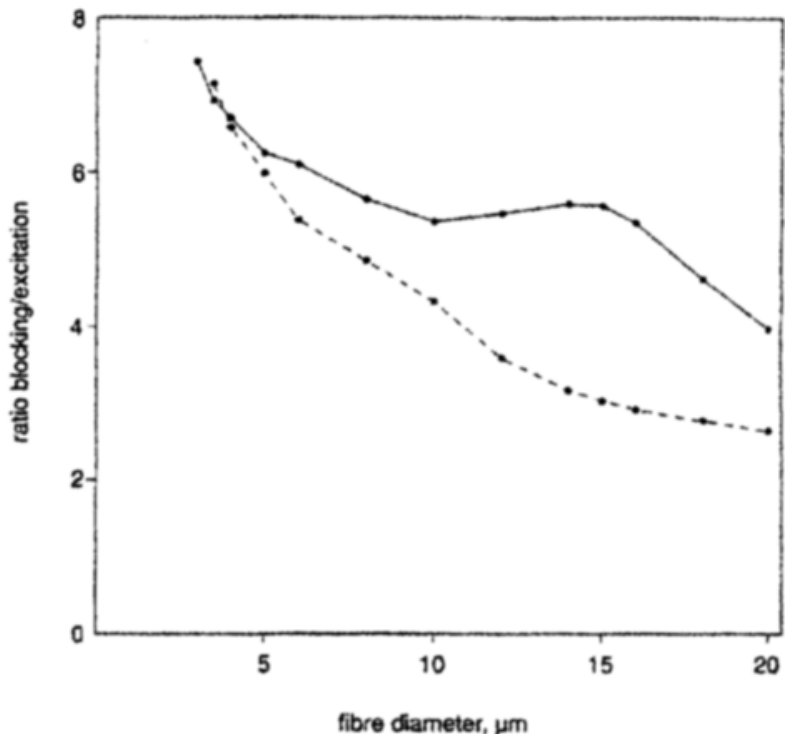

b

Fig. 4. (a) Excitation and blocking thresholds of a myelinated fibre as a function of fibre diameter for two electrode positions; a cathodal point source above a node (solid lines) and one-half of the internodal distance apart (dashed lines); pulse duration 300 ps; cathode-fibre distance $1 \mathrm{~mm}$; (b) ratio of blocking threshold to excitation threshold as a function of fibre diameter; pulse duration 300 us; cathodefibre distance $1 \mathrm{~mm}$; solid lines indicate cathode above a node of Ranvier; dashed lines indicate cathode centred between two neighbouring nodes

shaded area in Fig. $4 a$ indicates the minimum range for any fibre diameter resulting in excitation without blocking, independent of the cathode position with respect to the Ranvier nodes.

The blocking threshold is always higher than the threshold for excitation. The ratio of blocking threshold to excitation threshold depends on the electrode position and decreases with increasing fibre diameter. Fig. $4 b$ shows that if the cathode is centred between two neighbouring nodes (broken line), the ratio decreases gradually from 7.1 for $3.5 \mu \mathrm{m}$ fibre to 2.6 for $20 \mu \mathrm{m}$ fibre. When the cathode is above a node, the ratio does not continuously decrease with increasing fibre diameter. Between 10 and $15 \mu \mathrm{m}$ the ratio increases a little, but overall it decreases from 6.9 for $3.5 \mu \mathrm{m}$ fibre to 4.0 for $20 \mu \mathrm{m}$ fibre.

Fig. $4 a$ also shows the possibility of obtaining selective small fibre activation. Using a stimulus of $2.3 \mathrm{~mA}$, all fibres greater than $\sim 1.6 \mu \mathrm{m}$ are excited (point A), whereas all fibres above $\sim 5.8 \mu \mathrm{m}$ are blocked (point $\mathrm{B}$ ), thus resulting in the selective activation of all fibres between 1.6 and $5.8 \mu \mathrm{m}$ at a distance of $1.0 \mathrm{~mm}$ from the cathode.

\subsection{Relation between excitation/blocking threshold and elec- trode-fibre distance}

The nerve fibres in a nerve bundle are at different distances from the catbode. To assess the influence of the distance between fibre and cathode on the thresholds for excitation and blocking, we calculated the thresholds for a $5 \mu \mathrm{m}$ and $15 \mu \mathrm{m}$ fibre at various distances from the cathode. The thresholds have again been calculated for two different electrode positions:

(a) above a node of Ranvier

(b) centred between two neighbouring nodes.

The results (Fig. 5a) show that both excitation and blocking thresholds increase with increasing cathode-fibre distance.
Thus an increasing current would first excite and block the fibres closest to the electrode. Fibres at a larger distance from the electrode are only excited and blocked at higher current levels. The inftuence of the electrode position on the excitation threshold only decreases slightly with increasing electrodefibre distance. For example, increasing the distance from 0.4 to $1.8 \mathrm{~mm}$ reduces the difference in excitation threshold of a $15 \mu \mathrm{m}$ fibre between position (b) and position (a) from $0.106 \mathrm{~mA}$ to $0.101 \mathrm{~mA}$

Close to the cathode $(<0.7 \mathrm{~mm})$, the excitation threshold range of the $5 \mu \mathrm{m}$ fibre overlaps the excitation threshold range of the $15 \mu \mathrm{m}$ fibre, and selective activation of small fibres becomes accidental. At a distance of less than $0.5 \mathrm{~mm}$ the blocking thresholds of the $15 \mu \mathrm{m}$ fibre is very close to its excitation threshold.

The position of the cathode relative to the nodes strongly influences the blocking threshold. The electrode position that yields the lowest blocking threshold depends on the cathodefibre distance. From 0.4 to $0.8 \mathrm{~mm}$ the blocking threshold of the $15 \mu \mathrm{m}$ fibre is the lowest with the electrode above a node of Ranvier, whereas at distances of between 1.0 and $1.6 \mathrm{~mm}$ the threshold is lowest with the electrode centred between two neighbouring nodes.

The ratio between blocking threshold and excitation threshold increases with increasing electrode-fibre distance (Fig. $5 b$ ) and again there is a large relative influence of the electrode position with respect to the nodes of Ranvier.

Selective small fibre activation is only possible in a certain cathode-fibre distance range. Fig. $5 a$ shows the feasibility to activate $5 \mu \mathrm{m}$ fibres without activating $15 \mu \mathrm{mm}$ fibres in a range between $0.77 \mathrm{~mm}$ (point A) and $1.36 \mathrm{~mm}$ (point B) using a current of $1.5 \mathrm{~mA}$. At smaller distances the current is above the blocking threshold of $5 \mu \mathrm{m}$ fibres, whereas at larger distances the current is below the blocking threshold of the $15 \mu \mathrm{m}$ fibres. Beyond $1.73 \mathrm{~mm}$ the stimulus is not even sufficient to activate the $5 \mu \mathrm{m}$ fibres (point $\mathrm{C}$ ). At higher currents the distance range at which selective activation occurs increases. 

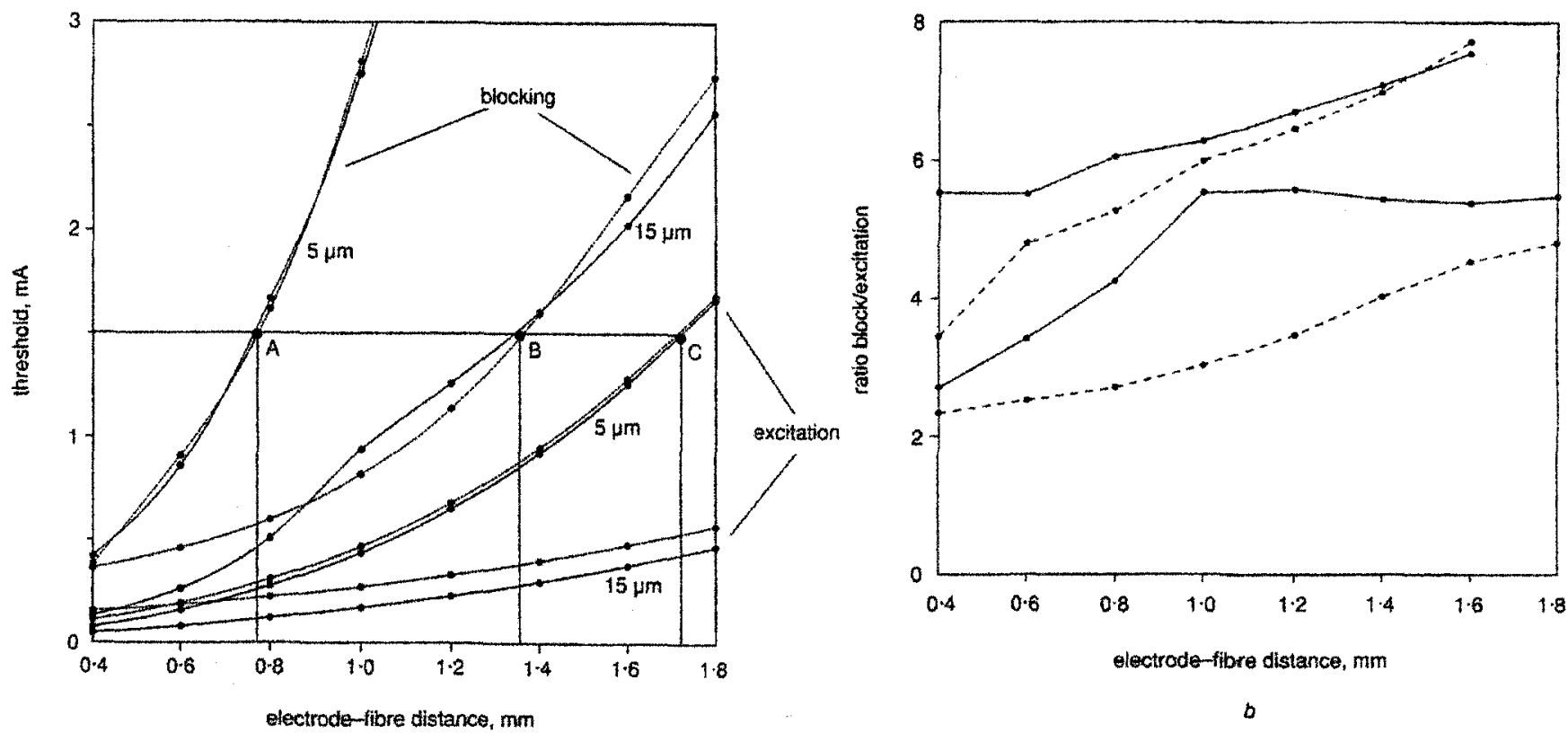

Fig. 5 (a) Excitation and blocking thresholds of $5 \mu \mathrm{m}$ and $15 \mu \mathrm{m}$ fibre as a finction of the electrode-fibre distance for two electrode positions. a cathode above a node (solid lines) and one-half of the internodal distance apart (dashed lines); pulse duration $300 \mu s ;(b)$ ratio of blocking threshold to excitation threshold as a function of the electrode-fibre distance; pulse duration $300 \mu$ s; solid lines indicate cathode above a node of Ranvier; dashed lines indicate cathode centred between two neighbouring nodes

\subsection{Relation between excitation/blocking threshold and pulse duration}

The propagation velocity of a nerve fibre is proportional to the fibre diameter. Therefore, if for each fibre diameter the most hyperpolarised node is at the same distance from the excitation point, it would take longer for an action potential of a small fibre to propagate from the excitation point to the hyperpolarised zone than for an action potential of a larger fibre. It may thus be possible to obtain a selective large fibre blockade by selecting the right pulse duration. Therefore, the influence of pulse duration on blocking threshold has been calculated for two fibre diameters (5 and $20 \mu \mathrm{m}$ ). As the position of the most hyperpolarised node depends on the cathode-fibre distance and the position of the cathode relative to the Ranvier nodes, the blocking thresholds have been calculated for two cathode-fibre distances $(0.6 \mathrm{~mm}$ and $1.4 \mathrm{~mm}$ ) and two electrode positions:

(a) above a node

(b) centred between two neighbouring nodes.

The relation between pulse duration on blocking threshold differs from the relation between pulse duration and excitation threshold. The reason is that blocking of an induced action potential at the minimum current must occur at the node with the greatest hyperpolarisation. This node is only a few nodes apart from the node that is first excited (Fig. 2). As it takes time for the action potential to propagate from the first excited node to the hyperpolarised node, there is a minimum pulse duration for blocking at low currents. Moreover, the hyperpolarising stimulus must stay at that node to compensate for the depolarising effect of the action potential at the neighbouring node, so long as the latter can cause depolarisation above the threshold. At smaller pulse durations blocking must occur at a node closer to the excited node, but due to a hyperpolarisation below the maximum value a higher current is necessary to block the action potential (RuKkHorf et al., 1994).

The lowest thresholds have been calculated with pulse durations above $250 \mu \mathrm{s}$. To compare the influence of the pulse duration on the blocking threshold, the calculated thresholds have been normalised by dividing them by the minimum threshold at $250 \mu \mathrm{s}$. When decreasing the pulse duration, the threshold remains almost constant until it suddenly rises sharply. Although in some configurations, the blocking thresholds show some discrete steps in the curves due to the previously explained effect of changing the blocking node (Fig. 6).

The pulse duration at which the sharp threshold increase occurs increases a little with decreasing fibre diameter, but is also influenced by the electrode position and the cathode-fibre distance. A different position can influence the minimal pulse duration to block a fibre at low currents by $60 \mu \mathrm{s}(5 \mu \mathrm{m}$ fibre at $1.4 \mathrm{~mm}$ distance). The resulting minimum pulse duration to block all fibres of a given diameter at minimum current is $235 \mu \mathrm{s}$ and $190 \mu \mathrm{s}$, for $5 \mu \mathrm{m}$ and $20 \mu \mathrm{m}$ fibre, respectively. When using a current level of 1.5 times the minimum blocking threshold, a minimum pulse duration of $130 \mu \mathrm{s}$ must be used to block all $20 \mu \mathrm{m}$. This coincides with the maximum pulse duration which can be used without blocking any $5 \mu \mathrm{m}$, regardless the current level. Thus pulse duration alone probably cannot be used as a parameter to obtain selective activation of small-diameter fibres.

\section{Discussion}

The aim of this study was to investigate the possibility of electrically activating small diameter myelinated nerve fibres without activating larger ones. Therefore, threshold currents have been calculated for excitation and blocking of nerve fibres with different diameters at different positions relative to the electrode.

The model to calculate the responses of myelinated nerve fibres to electrical stimulation consists of two parts that are not coupled. The first part is a volume conductor. In this part the electrical potentials external to the nodes of Ranvier imposed by the stimulus are calculated. These potentials are the input for the second part which is a myelinated nerve fibre model. A consequence of this method is that any influence is neglected 


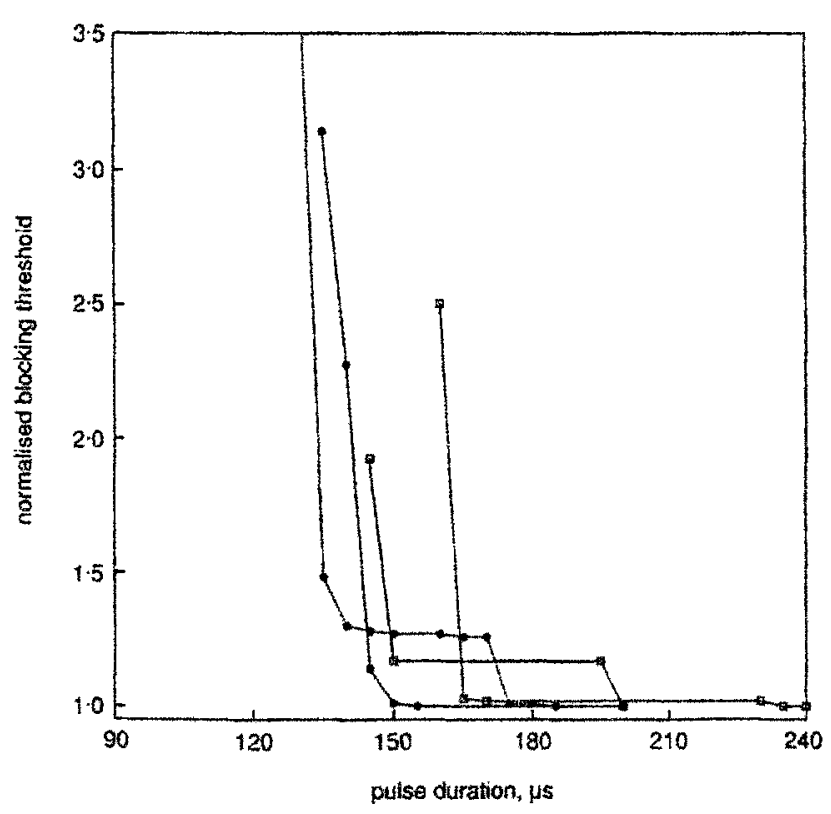

a

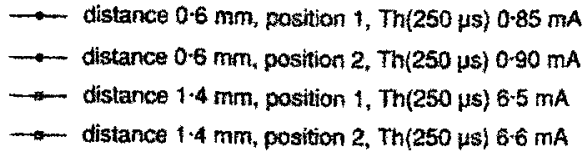

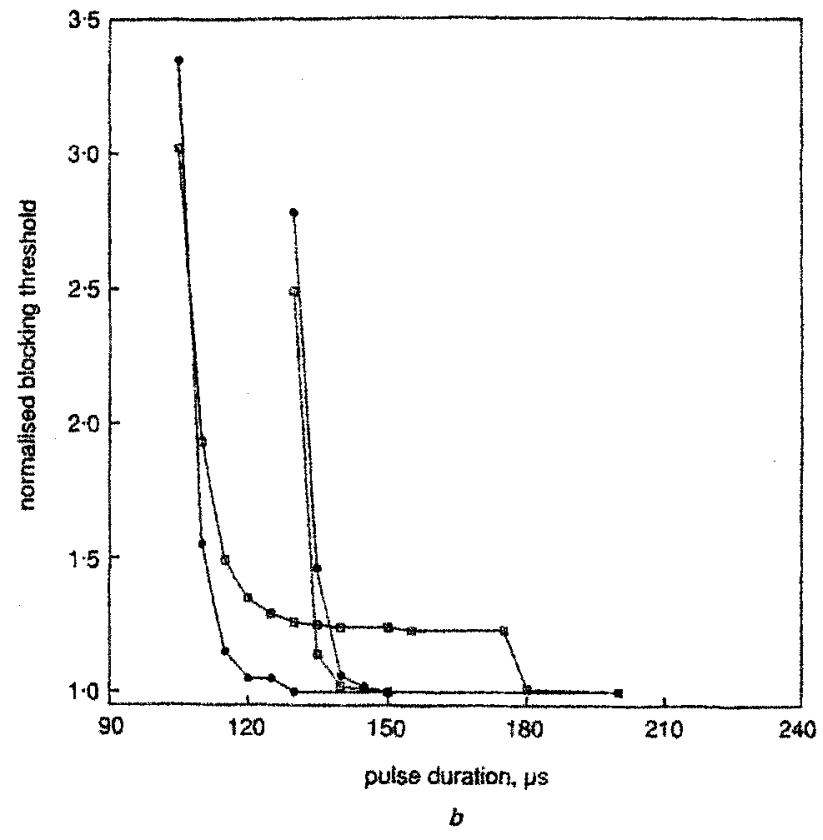

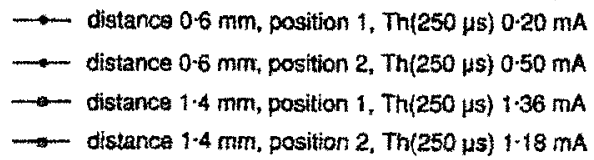

Fig. 6 Normalised blocking thresholds as a function of the pulse duration; thresholds have been normalised using the threshold at 250 us; distance $=$ cathode-fibre distance; position $1=$ cathode above a node; position $2=$ cathode centred between two neighbouring nodes; (a) 5 um fibre; (b) 20 um fibre

due to currents generated by the active fibres on the electric field imposed by the stimulation current. A significant infuence on the excitation thresholds is not expected, assuming that most fibres are inactive when a stimulus is given. However, blocking thresholds could be infuenced.

The isotropic and homogeneous volume conductor model is a simplified representation of the biological volume conductor. Anisotropy of nervous tissue and inhomogeneities such as blood vessels and connective tissue were not considered. These aspects can, however, be incorporated when using a more complex volume conductor model (ALTMAN and PLONSEY, 1988; VELTINK et al., 1988, 1989).

It is known from experiments on anodal blocking of peripheral nerves that fibre excitation can occur at the end of long-duration rectangular hyperpolarising pulses (VAN DEN HONERT and MORTIMER, 1981; FANG and MORTIMER, 1991a). This anodal break excitation phenomenon does not occur in the model. Hence, extending the pulse with a gradual decay (e.g. linear or exponential), as used experimentally to prevent excitation, was not necessary in the simulations.

The stimuli used in the study were monophasic current pulses. In chronic implants, however, monophasic pulses might induce neural damage and electrode contact corrosion due to a net transfer of a charge at the contact. A secondary reversed pulse must be added to reduce the net injected charge. In tripolar stimulation this is a problem, because the anodes become cathodic and may cause excitation if the threshold is exceeded. In monopolar stimulation this is less of a problem, because the cathode becomes anodic and more current is required from an anode than from a cathode to excite an axon (RANCK, 1975).

The calculated relation between excitation/blocking threshold and fibre diameter (Fig. 4a) shows the possibility of achieving selective small fibre activation. Unfortunately, there is a large relative influence of the cathode-fibre distance. This prevents a uniform selective activation of small fibres in the whole nerve bundle and limits it to a region some distance from the cathode. The region is limited because small fibres may be blocked close to the cathode whereas at greater distances either the large fibres are not blocked or the small fibres are not activated. The influence of the cathode-fibre distance will, however, be reduced when using a small ring electrode around the nerve bundle instead of a point source.

Only a few quantitative experimental data on monopolar blocking, useful for a comparison with our theoretical results, have been published. Roberts and Smith reported a ratio of blocking threshold to excitation threshold of $8.3 \pm 2.7$ (mean $\pm S D$ ) when stimulating fibres in the spinal cord of a cat using a pulse duration of $0.1 \mathrm{~ms}$ (ROBERTs and SMTTH, 1973). However, absolute thresholds, fibre diameters and electrode-fibre distances have not been reported. Their blocking/excitation ratio is, however, a little higher than our model predicts (Fig. $5 b$ ). Surprisingly they obtained blocking using a $0.1 \mathrm{~ms}$ pulse duration which is less than the minimum pulse duration predicted for a $20 \mu \mathrm{m}$ fibre.

Tai and Jiang demonstrated blocking effects of large fibres in a toad's sciatic nerve at $18.5^{\circ} \mathrm{C}$; however they needed $20 \mathrm{~mA}$ to obtain blocking (TAI and JIANG, 1994). This high current was probably because the anode was positioned only $2.5 \mathrm{~cm}$ from the cathode, opposite the place where blocking was intended. This probably reduced the hyperpolanising effect at the intended blocking site.

Dreyer et al. described how they failed to obtain blocking when stimulating the median and superficial radial nerves using monopolar surface electrodes in humans (DREYER, et al., 1993). In their configuration the distance between electrode and nerve is more than $2 \mathrm{~mm}$. Our model predicts a ratio between excitation and blocking of more than 4 for this distance (Fig. 5b). Measurements of the evoked compound action potentials showed a maximum response at a cathodal 
stimulus of $22.7 \mathrm{~mA}$ (mean), and so blocking should occur around $90 \mathrm{~mA}$. However, the stimulator of Dreyer et al. was limited to $80 \mathrm{~mA}$, which is below the blocking threshold and therefore they did not obtain blocking effects.

Our theoretical results show the feasibility of obtaining selective small fibre activation using a single cathode. Although at least two electrode contacts are necessary, stimulation is considered monopolar if only the cathode is close to the target nerve bundle. The second contact (anode) is located at some distance from the cathode. It is possible that the current flow from the cathode to the anode excites other excitable tissue around the electrodes. Although this side-effect can be reduced by employing an anode with relatively large dimensions, it could limit the clinical application of this method.

Acknowledgment-This work has been supported by the Dutch Kidney Foundation C92.1249.

\section{References}

ACCORNERO, N., BNI, G., LENZI, G. L., and MANFREDI, M. (1977): 'Selective activation of peripheral nerve fibre groups of different diameter by triangular shaped stimulus pulses', J. Physiol., 273, pp. $539-560$

ALTMAN, K. W., and PLONSEY, R. (1988): 'Development of a model for point source electrical fibre bundle stimulation', Med. Biol. Eng. Comput., 26, pp. 466-475

BLAIR, E., and ERLANGER, J. (1933): 'A comparison of the characteristics of axons through their individual electrical responses', Am. J. Physiol., 106, pp. 524-564

BRISMAR, T. (1980): 'Potential clamp analysis of membrane currents in rat myelinated nerve fibres', J. Physiol, 298, pp. 171-184

ChU, S. Y., RITCHE, J. M., ROGART, R. B., and STAGG, D. (1979): 'A quantitative description of membrane currents in rabbit myelinated nerve', J. Physiol, 292, pp. 149-166

Dreyar, S. J., DuMTrRU, D., and KNG, K. C. (1993): 'Anodal block v anodal stimulation. Fact or Fiction', Am. J. Phys. Med. Rehabil., 71, pp. 10-18

FANG, Z.-P, and MORTMMER, J. T. (1991a): "Selective activation of small motor axons by quasitrapezoidal current pulses', IEEE Trans., BME-38, pp. 168-174

FANG, Z.-P., and MORTMER, J. T. (1991b): 'A method to effect physiological necruitment order in electrically activated muscle', IEEE Trans., BME-38, pp. 175-179

GEDDES, L. A, and BAKER, L. E. (1967): "The specific resistance of biological material-a compendium of data for the biomedical engineer and physiologist', Med. Biol. Eng., 5, pp. 271-293

HENNEMAN, E. (1981): 'Recruitment of motoneurons: the size principle in DESMEDT, J. E. (Ed): Prog. Clin. Neurophysiol., Karger, Basel, 9, pp. 26-60

VAN DEN HONERT, C., and MORTMER, J. T. (1981): 'A technique for collision block of peripheral nerve: single stimulus analysis", IEEE Trans., BME-28, pp. 373-378

MCNEAL, D. R. (1976): Analysis of a model for excitation of myelinated nerve', IEEE Trans., BME-23, pp. 329-337

RANCK, 3. B. (1975): "Which elements are excited in electrical stimulation of mammalian central nervous system: a review', Brain Res., 98, pp. 417-440

RATTAY, F. (1987): "Ways to approximate current-distance relations for electrically stimulated fibres', J. Theor. Biol, 125, pp. 339-349

RATTAY, F. (1989): 'Analysis of models for extracellular fibre stimulation', IEEE Trans., BME-36, pp. 676-682

RIIKHOF, N. J. M., KOLDEWIN, E. L., VAN KRRREBROECK, P. E. V, DusRUYnE, F. M. J., and WoKsTRA, H. (1993): 'Selective activation of the detrusor muscle by sacral root stimulation in a canine model', Netcrourol. Urodyn., 12, pp. 381-382
RIKHOFF, N. J. M., HOLSHEIMER, J., KOLDEWIJN, E. L., STRLIJ, J. J., VAN KERREBROECK, P. E. V., DEBRUYNE, F. M. J., and WUKSTRA, H. (1994): 'Selective stimulation of sacral nerve roots for bladder control: a study by computer modeling', IEEE Trans., BME-41, pp. $413-424$

ROBERTS, W. J., and SMTTH, D. O. (1973): 'Analysis of threshold currents during microstimulation of fibres in the spinal cord', Acta Physiol. Scand. 89, pp. 384-394

SCHALOW, G., and BARTH, H. (1992): 'Group conduction velocities and nerve fibre diameters of $\alpha$ and $\gamma$-motoneurons from lower sacral nerve roots of the dog and humans', Gen. Physiol. Bioph., 11, pp. $85-99$

SCHMIDT, R. A. (1986): 'Advances in genitourinary neurostimulation', Neurosurg., 19, pp. 1041-1044

SOLOMONOW, M. (1984): 'External control of the neuromuscular system', IEEE Trans., BME-31, pp. 752-763

STRUUK, J. J., HOLSHEIMER, J., VAN DER HEIDE, G. G., and BOOM, H. B. K. (1992): 'Recruitment of dorsal column fibres in spinal cord stimulation: influence of collateral branching', IEEE Trans, BME39 , p. $903-912$

TAI, C., and JIANG, D. (1994): 'Selective stimulation of smaller fibres in a compound nerve trunk with single cathode by rectangular current pulses", IEEE Trans., BME-41, pp. 286-291

VELTTNK, P., VAN ALSTE, J. A., and BOOM, H. B. K. (1988): 'Simulation of intrafascicular and extraneural nerve stimulation', IEEE Trans., BME-35, pp. 69-75

VELTINK, P., VAN VEEN, B. K., STRUYJK, J. J, HOLSHEIMER, J, and BOOM, H. B. K. (1989): 'A modeling study of nerve fascicle stimulation', IEEE Trans., BME-36, pp. 683-692

\section{Appendix: list of symbols}

$C_{m}=$ nodal membrane capacity, $\mathrm{F}$

$R_{m}=$ nodal membrane resistance, $\Omega$

$R_{a}=$ intra-axonal resistance, $\Omega$

$V_{\text {rest }}=$ transmembrane resting potential, $\mathrm{V}$

$V_{i, n}=$ intracellular potential at node $n, V$

$V_{e, n}=$ extracellular potential at node $n, \mathrm{~V}$

$V_{n}=$ reduced transmembrane potential, $\mathrm{V}$

$i_{i o n, n}=$ ionic current density through nodal membrane at node $n, \mathrm{~A} \mathrm{~m}^{-2}$

$D=$ fibre diameter, $\mathrm{m}$

$d=$ axon diameter, $\mathrm{m}$

$L=$ internodal distance, $\mathrm{m}$

$t=$ time, $s$

$c_{m}=$ nodal membrane capacitance, $0.02 \mathrm{~F} \mathrm{~m}^{-2}$

$\rho_{a}=$ axoplasm resistivity, $0.7 \Omega \mathrm{m}$

$l=$ nodal length, $1.5 \mu \mathrm{m}$

$d D=$ ratio of axon to outer fibre diameter, 0.6

$L L D=$ ratio of intemodal distance to fibre diameter, 100

\section{Author's biography}

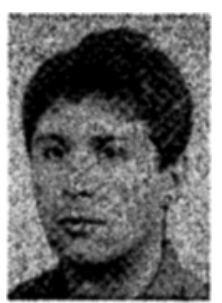

Nico J. M. Rijhof was bom in Opmeer, The Netherlands, in 1964. He recerved his MSc in Electrical and Biomedical Engineering in 1990 from the University of Twente, Enschede, The Netherlands. Since 1991 he has been working towards his PhD at the Biomedical Engineering Group of the Department of Urology at the University Hospital Nijmegen. His research interests are restoration of urinary bladder control in spinal cord injury by electrical stimulation of the sacral nerve roots. 